\title{
Gelation of Gelatin Solution
}

\author{
Akira HAYASHI and Shin-Chol OH \\ Department of Chemistry, Ritsumeikan University, \\ Tojiinkita-machi, Kita-ku, Kyoto 603, Japan
}

Received August 23, 1982

\begin{abstract}
In order to study the gelling mechanism of gelatin, the phase diagram for a diluted gelatin solution was investigated. Phase boundary curves were obtained by measuring the fluorescent depolarization, optical rotation, reduced viscosity, and light scattering of the gelatin solution. The phase diagram obtained exhibited similar features to those for amylose and agarose gelling systems. From this similarity, the gelling mechanism of gelatin is concluded to be as follows: The randomly coiled gelatin molecule in hot solution partially recovers a collagen fold at the denaturation temperature near $40^{\circ} \mathrm{C}$ on cooling. This gelatin state is presumed to correspond to the interrupted helical form for amylose. This partial helical solution then separates into two phases, a dilute solution and a coacervate consisting of a concentrated solution, at a phase separation temperature which depends upon the gelatin concentration. When the concentration is sufficiently high, coacervate particles are connected with each other to form a three dimensional macroscopic network, and the solution loses its fluidity to become a gel.
\end{abstract}

The collagen molecule in hot solution takes a randomly coiled structure designated as gelatin and partially recovers its helical structure, the so-called collagen fold, on cooling. When the concentration is sufficiently high, the solution loses its fluidity to form a gel at a lower temperature. Several mechanisms for this gelation have been proposed. ${ }^{1)}$ These mechanisms have usually been advanced on the basis of a comparison of the solution properties between a denaturated and a gelled solution, or of kinetic studies of solution property changes at a temperature sufficiently below the denaturation point. The most common conclusion is that gelation results from the aggregation or crystallization of collagen folds.

The examination of this literature on gelation indicates that the gelation does not gel at the denaturation or helix-coil transition temperature, but gels at a certain temperature sufficiently below the denaturation point. For example, the optical rotation data of Flory et $a .^{2)}$ shows an additional abrupt change, which is supposed to be the gel point, at a lower temperature than the denaturation point and the light scattering data of Doty et al. ${ }^{3)}$ also suggests the existence of a certain critical gelling temperature, although these authors seem to attach no importance to the existence of this temperature.

Recently, Eagland et al. $^{4)}$ proposed that the gelation is caused by the van der Waals-London dispersion attractive force bctween the hydrated shells of a partial collagen folded molecule. They calculated the relationship between gelatin concentration and the gelling temperature at which the first contact between hydrated shells occurs in solution, and showed that the actual gelling temperature observed was coincident with this calculation.

In our laboratory, the gelling mechanism of $\operatorname{agarose}^{5 \sim 7)}$ and amylose solutions ${ }^{8)}$ have been studied and it was found that when hot solutions of these polymers were cooled, the polymers in solution were partially renaturated to an interrupted helical structure followed by separation into two solution phases. The phase separation temperature was apparently lower than the helix-coil transition point and depended upon the concentration of the polymers. If the concentration was suf- 
ficiently high, the solution gelled at the phase separation temperature. This meant that the gelation was not caused directly by the partial helix formation itself but by the phase separation. From this result, it was concluded that gelation occurred with a network formation among the coacervate particles in the dilute solution phase.

Against this background, the present authors wondered if the gelation of gelatin solution was also caused by a mechanism similar to agarose or amylose. In order to resolve our question, the relationship between critical temperatures concerned with gelation and gelatin concentration was determined by the various methods employed in preceding papers for agarose and amylose.

\section{MATERIALS AND METHODS}

The collagen sample for the gelatin solution was 'Koken Atelocollagen' (Koken Co. Ltd, Tokyo, Japan). ${ }^{9)}$ This sample is a soluble, telopeptide-free calf skin collagen treated by a protease, and, therefore, yields a pure $\alpha$-gelatin solution free from telopeptide when the solution is heated above the denaturation temperature. The intrinsic viscosity is $16 \sim 18 \mathrm{dl} / \mathrm{g}(\mathrm{MW}=300,000$ as a triple helix) in dil. $\mathrm{HCl}$ solution of $\mathrm{pH} 3$, and the denaturation temperature is $38.6^{\circ} \mathrm{C}$

The gelatin solution was prepared by heating the collagen suspended in water for $2 \mathrm{hr}$ at $55^{\circ} \mathrm{C}$, with $0.1 \%$ methylparaben as an antiseptic. For measurements of the fluorescent depolarization with an extrinsic probe, the solutions contained $10^{-5} \mathrm{M}$ uranine. In this case, it was ascertained that the fluorescent dye, uranine, was adsorbed on the gelatin molecule. The concentration of each gelatin solution was adjusted at the beginning of preparation, to guard against varying concentration, since it is known that the successive dilution of a solution at temperatures below the denaturation point causes a result different from the direct preparation as shown by Doty et $a l .{ }^{3)}$ The setting time for a given temperature before measurements is shown in each result.

The instruments used were a Hitachi Fluorescence Spectrophotometer 650-10S with an attachment for fluorescent polarization, a Union Automatic Digital Polarimeter PM-101, and an Ubbelohde-type viscometer, to measure the fluorescent depolarization $(1 / p)$, optical rotation at a wavelength of $405 \mathrm{~nm}\left([\alpha]_{405}\right)$, and the reduced viscosity $\left(\eta_{\mathrm{sp}} / c\right)$, respectively. Details of these measurement methods were described in previous papers. $^{5 \sim 8)}$

As a simple measurement of gelatin particle size in solution, the qualitative light scattering at $90^{\circ}\left(i_{90}\right)$ was measured with the Hitachi Fluorescence Spectrophotometer $650-10 \mathrm{~s}$. The wavelength used was $500 \mathrm{~nm}$ for both incident and scattered light. The sensitivity of instrument was first set so that the $i_{90}$ of .water showed zero, and then the $i_{90}$ of the sample solution was measured at this sensitivity. The result is expressed as $c / i_{90}$, a qualitative reduced intensity of scattering, where $c$ was the gelatin concentration.

\section{RESULTS}

Figure 1 shows $1 / p$ for $0.3,0.8$, and $1.2 \mathrm{~g} / \mathrm{dl}$ gelatin solutions as a function of temperature when the hot solution is cooled slowly. The setting time for each temperature was $1 \mathrm{hr}$ for temperatures above $36^{\circ} \mathrm{C}$ and $6 \sim 12 \mathrm{hr}$ below $35^{\circ} \mathrm{C}$. These curves in the figure are only three typical examples among many experiments for varying concentration. The $1 / p$ in each curve shows an abrupt change near $40^{\circ} \mathrm{C}$. This temperature does not change much with the concentration and corresponds to the denaturation point or helix-coil transition point. There seems to be another abrupt change at a temperature below $30^{\circ} \mathrm{C}$. This second temperature depends upon the concentration of the polymer and the concentration dependence becomes larger as the concentration is decreased. By observation with the naked eye, gelation was recognized to occur at this temperature in concentrated solutions above about $0.5 \mathrm{~g} / \mathrm{dl}$.

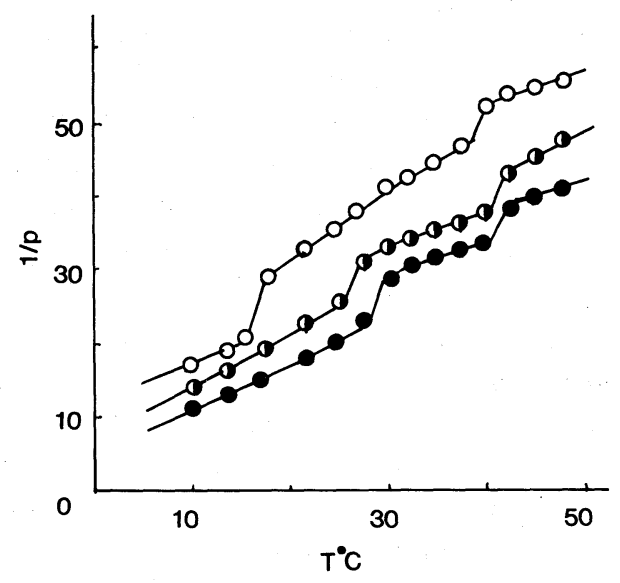

FIG. 1. Temperature Dependence of the Fluorescent Depolarization $(1 / p)$ for $0.4(\bigcirc), 0.8(\odot)$, and $1.2 \mathrm{~g} / \mathrm{dl}(\bullet)$ Gelatin Solutions Containing $10^{-5} \mathrm{M}$ Uranine. 


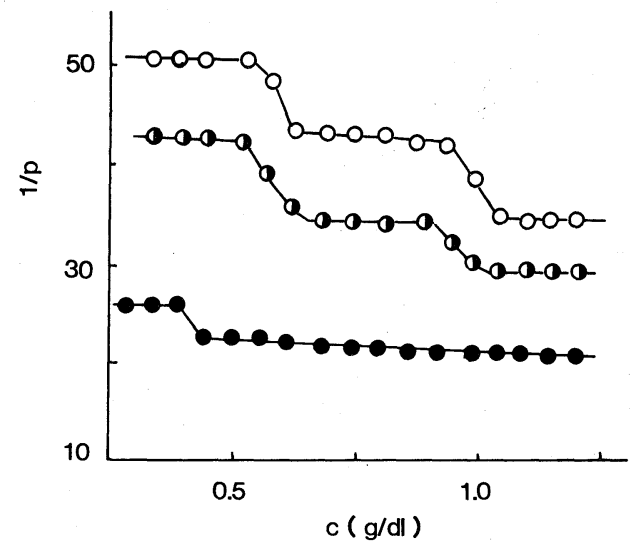

FIG. 2. Concentration Dependence of the Fluorescent Depolarization at $34(\mathrm{O}), 30(\mathbf{O})$, and $23^{\circ} \mathrm{C}(\mathbf{O})$.

Figure 2 shows $1 / p$ at 23,30 , and $34^{\circ} \mathrm{C}$ as a function of concentration. These solutions were stood at given temperatures for $24 \mathrm{hr}$ before measurements. These curves are also only typical examples among many experiments. The $1 / p$ at $30 \sim 40^{\circ} \mathrm{C}$ shows two abrupt changes at about 0.5 and $1.0 \mathrm{~g} / \mathrm{dl}$. There seems no abrupt change at temperatures over $45^{\circ} \mathrm{C}$, although they are not cited here in the figure. The $1 / p$ at temperatures below about $28^{\circ} \mathrm{C}$ shows no abrupt change at 0.5 and $1.0 \mathrm{~g} / \mathrm{dl}$, but shows, instead, another abrupt change at a concentration lower than $0.5 \mathrm{~g} / \mathrm{dl}$. This concentration becomes lower as the temperature is decreased.

Figure 3 shows $[\alpha]_{405}$ at 24,30 , and $34^{\circ} \mathrm{C}$ as a function of concentration. The conditions for measurement were the same as in the case of Fig. 2. The $[\alpha]_{405}$ at $30 \sim 40^{\circ} \mathrm{C}$ shows two troughs or peaks at nearly the same concentration as $1 / p$ in Fig. 2, about 0.5 and 1.0 $\mathrm{g} / \mathrm{dl}$. The $[\alpha]_{405}$ at $24^{\circ} \mathrm{C}$ shows two similar troughs at 0.5 and $1.0 \mathrm{~g} / \mathrm{dl}$, and, additionally, another reverse trough which bottoms out at a concentration lower than $0.5 \mathrm{~g} / \mathrm{dl}$ and corresponds to that in $1 / p$ at $23^{\circ} \mathrm{C}$ in Fig. 2.

Figure 4 shows $\eta_{\mathrm{sp}} / c$ for 0.3 and $1.2 \mathrm{~g} / \mathrm{dl}$ solutions as a function of temperature when the hot solution is slowly cooled. The setting time for each temperature was $8 \sim 12 \mathrm{hrs}$ before measurements. The $\eta_{\mathrm{sp}} / c$ in both concentrations shows a small trough, which is re-

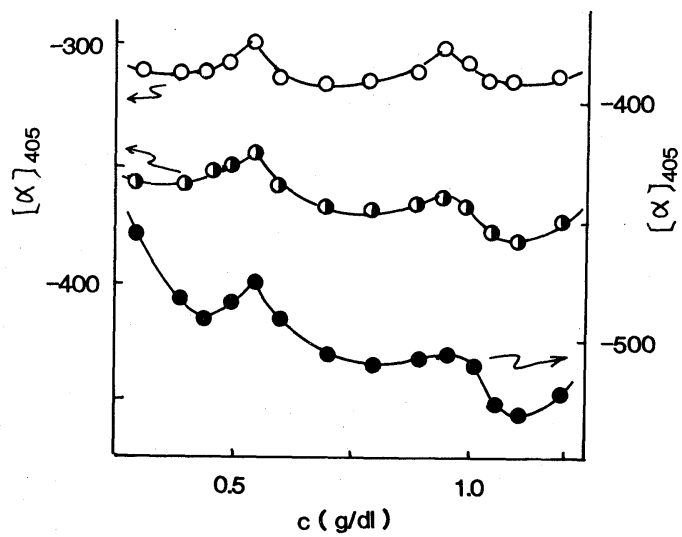

FIG. 3. Concentration Dependence of the Specific Optical Rotation at $405 \mathrm{~nm}$ Wavelength $\left([\alpha]_{405}\right)$ at $34(\mathrm{O})$, 30 (O), and $24^{\circ} \mathrm{C}(\odot)$.

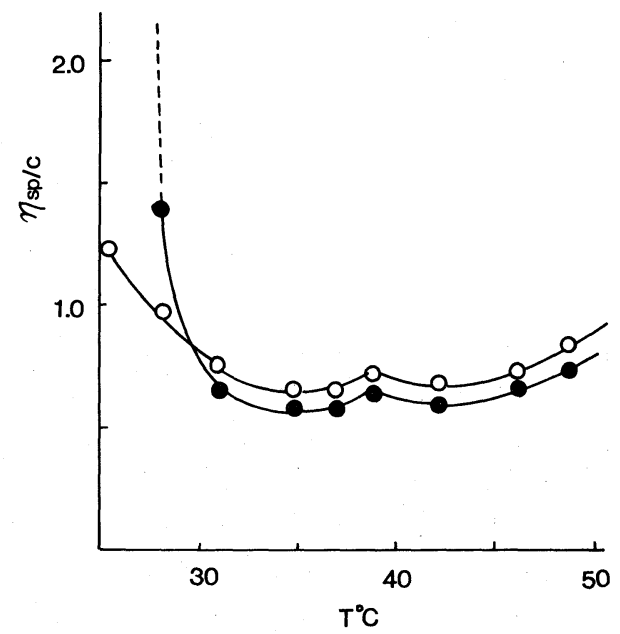

Fig. 4. Temperature Dependence of the Reduced Viscosity $\left(\eta_{\mathrm{sp}} / c\right)$ for $0.3(\bigcirc)$ and $1.2 \mathrm{~g} / \mathrm{dl}(\bigcirc)$ Gelatin Solutions.

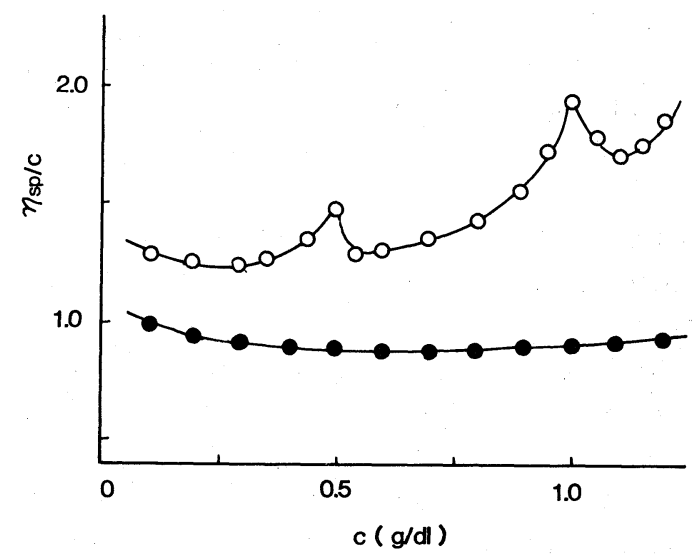

FIG. 5. Concentration Dependence of the Reduced Viscosity at $34(\bigcirc)$ and $50^{\circ} \mathrm{C}(\odot)$. 


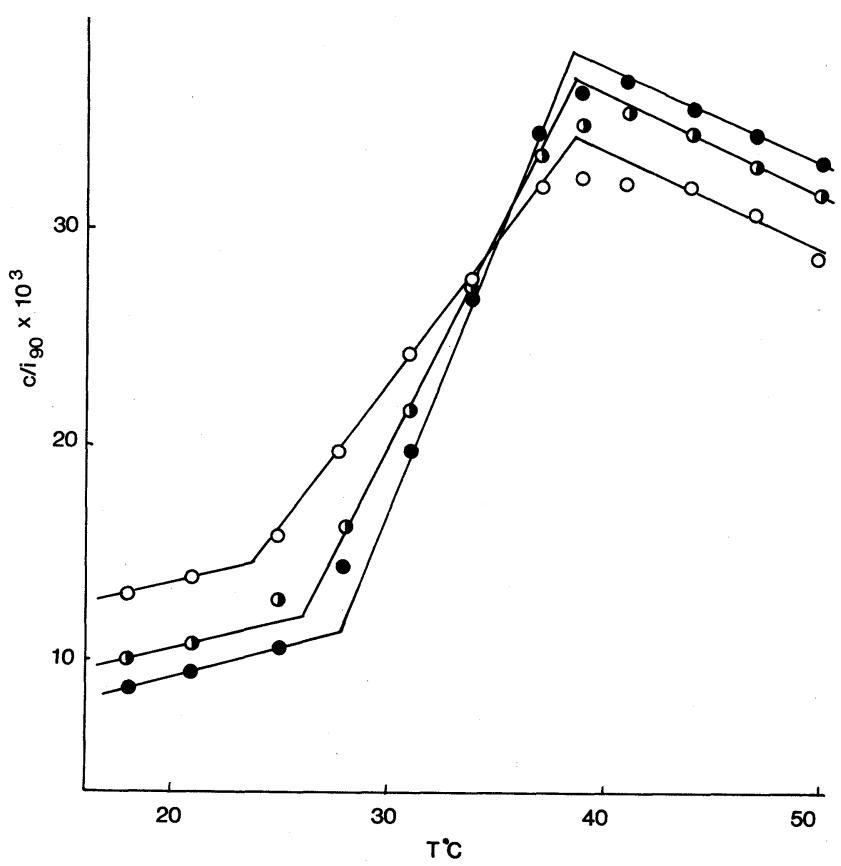

FIG. 6. Temperature Dependence of the Reduced Intensity of Light Scattering $\left(c / i_{90}\right)$ for $0.4(O), 0.8(O)$, and $1.2 \mathrm{~g} / \mathrm{dl}(\mathbf{O})$ Gelatin Solutions.

garded to be a crossing point of two different curves, at about $40^{\circ} \mathrm{C}$ corresponding to the denaturation point. The rapid increase in $\eta_{\mathrm{sp}} / c$ for $1.2 \mathrm{~g} / \mathrm{dl}$ solution near $28^{\circ} \mathrm{C}$ shows gelation of the solution. The measurable value of $\eta_{\mathrm{sp}} / c$ for the $0.3 \mathrm{~g} / \mathrm{dl}$ solution fluctuated and became poor in its reproducibility at temperatures below about $20^{\circ} \mathrm{C}$, indicating that the solution became heterogeneous but did not gel. The value of $\eta_{\text {sp }} / c$ for the $0.3 \mathrm{~g} / \mathrm{dl}$ solution is slightly higher than that for $1.2 \mathrm{~g} / \mathrm{dl}$ at temperatures over $30^{\circ} \mathrm{C}$. This is due to the polyelectrolytic expansion of the gelatin molecule as shown by the next experiment in Fig. 5.

Figure 5 shows $\eta_{\text {sp }} / c$ at 34 and $50^{\circ} \mathrm{C}$ as a function of concentration. These solutions were stood for $2 \sim 3 \mathrm{hr}$ at given temperatures before measurements. The $\eta_{\mathrm{sp}} / c$ at $34^{\circ} \mathrm{C}$ shows two distinct troughs at about 0.5 and $1.0 \mathrm{~g} / \mathrm{dl}$ and similar to the cases of $1 / p$ and $[\alpha]_{405}$. The $\eta_{\mathrm{sp}} / c$ at $50^{\circ} \mathrm{C}$ does not show any detectable change because of the randomly coiled conformation. The slight increase in $\eta_{\mathrm{sp}} / c$ at lower concentrations is due to a weak polyelectro-

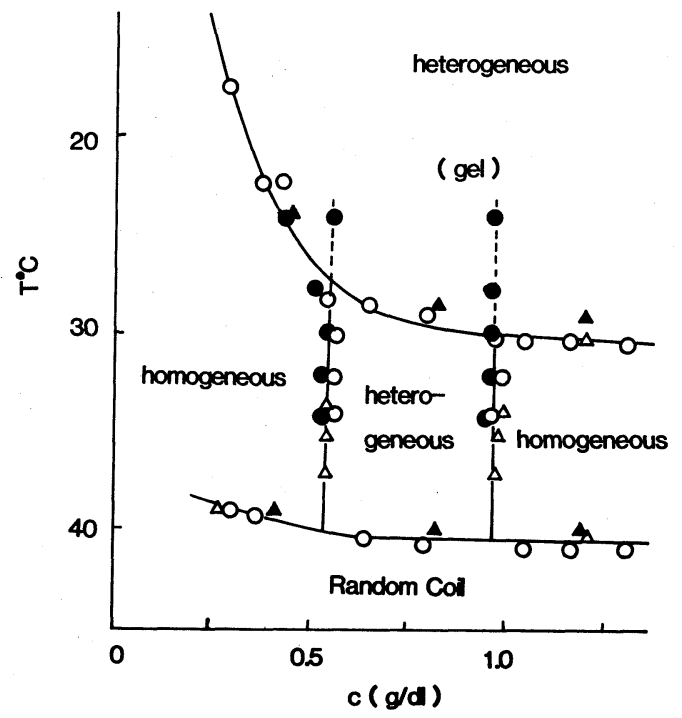

FIG. 7. Phase Diagram for Gelatin Solution.

$\bigcirc, 1 / p ; \bigcirc,[\alpha]_{405} ; \Delta, \eta_{\mathrm{sp}} / c ; \Delta, c / i_{90}$.

lytic expansion in the $0.1 \%$ methylparaben solution.

Figure 6 shows $c / i_{90}$ for $0.4,0.8$, and $1.2 \mathrm{~g} / \mathrm{dl}$ solutions as a function of temperature when the hot solution is slowly cooled. The setting 
time for a given temperature was $1 \mathrm{hr}$ for temperatures above $36^{\circ} \mathrm{C}$ and $6 \sim 12 \mathrm{hr}$ below $35^{\circ} \mathrm{C}$. All curves show a discontinuity, corresponding to the helix-coil transition point, at about $40^{\circ} \mathrm{C}$ and a second discontinuity corresponding to the case of $1 / p$ in Fig. 1 .

Figure 7 shows the relationship between polymer concentration and the temperature at which an apparent change is observed in solution properties as shown in Figs. $1 \sim 6$. In this figure, some related data which are not drawn in Figs. $1 \sim 6$ are also contained. The solid line connecting each point in the figure shows a characteristic pattern similar to those for agarose, ${ }^{7)}$ amylose, ${ }^{8)}$ and an amylosebutanol complex. ${ }^{10}$ ) The dotted line and the interpretation of the solution phase in the figure will be explained later in discussion.

\section{DISCUSSION}

The solution properties of $\alpha$-gelatin solution prepared from Atelocollagen were measured as shown in Figs. $1 \sim 6$. These data undoubtedly reflect the molecular behavior of $\alpha$-gelatin in solution but some unknown factors still remained to be resolved before we could determine the gelatin conformation from these data. In the present work, the purpose of the measurements is not for the direct determination of gelatin conformation itself, but for drawing the phase diagram from changes in the solution properties. For this purpose, the measurement of $1 / p$ with uranine as an extrinsic probe have been shown to be an effective method as seen in our previous papers. ${ }^{5 \sim 8)}$ The results from $1 / p$ measurement in Figs. 1 and 2 agree well with those from other methods in Figs. $3 \sim 6$. The phase diagram in Fig. 7 is drawn from these results. This diagram basically resembles those for the agarose and amylose gelling systems.

There seems no basis so far that the gelation of protein solution occurs with the same mechanism as carbohydrate solution, but the diagram in Fig. 7 suggests that the gelling mechanism of gelatin is a similar type to that of carbohydrate.
It is apparent that the solid line near $40^{\circ} \mathrm{C}$ in Fig. 7 is the coil-helix transition or denaturation line. The precise conformation of gelatin at temperatures below the denaturation point is still in doubt but the existence of an interrupted helical form is not so difficult to imagine. Therefore, it is possible to assume that an interrupted helical structure of $\alpha$-gelatin is produced in the region above the helix-coil transition line. This interrupted helical solution gels at a certain temperature near $30^{\circ} \mathrm{C}$ when the concentration is higher than $0.5 \mathrm{~g} / \mathrm{dl}$. This gelling temperature is apparently lower than the denaturation point and depends slightly upon the concentration. Solutions more diluted than $0.5 \mathrm{~g} / \mathrm{dl}$ make no gel but show a certain critical temperature at which the solution properties are abruptly changed and their measurable values fluctuate slightly as shown in the experiments. This temperature apparently depends upon the concentration more than the gelling point. It is recognized that this critical temperature and the gelling temperature are both on an identical curve line as seen in Fig. 7. From these results and a similarity to agarose or amylose, it is also recognized that this curve line corresponds to the phase line separating into two phases, a dilute solution phase and a coacervate, consisting of conc. solution, phase. The $c / i_{90}$ in Fig. 6 is just a qualitative datum. However, the sharp discontinuity of $c / i_{90}$ at the temperature discussed here shows a somewhat serious phase change and may reflect the phase separation.

The region partitioned by vertical lines of about 0.5 and $1.0 \mathrm{~g} / \mathrm{dl}$, and by transverse lines of about 30 and $40^{\circ} \mathrm{C}$ in Fig. 7 was detected clearly by changes in the solution properties as shown in our experimental data, but the detection of heterogeneity was very difficult compared with the cases for agarose and amylose. A similar difficulty in the detection of heterogeneity was also observed in the heterogeneous phase at temperatures lower than the phase separation point. This may be due to poor content of the helical segment in the interrupted helix of gelatin compared with that of 
agarose or amylose, but there is no apparent evidence for it and this is just an assumption.

The common phase diagram among gelatin, agarose, and amylose apparently resembles, in pattern, that of rod-like polymer solution, ${ }^{11)}$ and the interpretation of the gelling mechanism with an interrupted helix formation followed by a phase separation is tacitly based upon the theory for rod-like polymers. However, the relationship between a rod-like polymer and an interrupted helical polymer is still not very clear and cannot be discussed here in more detail, although further examination continues.

The dotted lines in Fig. 7 are detectable only with measurements of some solution properties. The most apparent example is the $[\alpha]_{405}$ at $24^{\circ} \mathrm{C}$ in Fig. 3 and a similar phenomenon was also observed in the case of agarose. The reason why there seems such a change in the heterogeneous, separated phase is suggested as follows: The solution containing less gelatin than $0.5 \mathrm{~g} / \mathrm{dl}$ or more than $1.0 \mathrm{~g} / \mathrm{dl}$ is a homogeneous helical solution until phase separation occurs, and it changes firstly into a heterogeneous solution when the temperature reaches the phase separation point. On the other hand, the solution containing $0.5 \sim 1.0$ $\mathrm{g} / \mathrm{dl}$ of gelatin is already a heterogeneous helical solution at the denaturation temperature. This heterogeneous solution changes to another heterogeneous solution at the phase separation point. All phase-separated solutions are expected to be of a similar type and no dotted line is expected to be seen if the system reaches final equilibrium. However, it may take so long to reach equilibrium that the difference in history of each helical solution may remain as the dotted line. As this interpretation shows, the phase separation line between 0.5 and $1.0 \mathrm{~g} / \mathrm{dl}$ is obviously detectable as a boundary between one heterogeneous solution and another heterogeneous solution, although the corresponding line is not seen in Flory's phase diagram for rodlike polymer solutions. ${ }^{11)}$

\section{REFERENCES}

1) A. Veis, "The Macromolecular Chemistry of Gelatin," Academic Press, New York, 1964.

2) P. J. Flory and E. S. Weaver, J. Am. Chem. Soc., 82, 4518 (1960).

3) H. Boedtker and P. Doty, J. Phys. Chem., 58, 968 (1964).

4) D. Eagland, G. Pilling and R. G. Wheeler, Faraday Discuss. Chem. Soc., 57, 181 (1974).

5) A. Hayashi, K. Kinoshita and M. Kuwano, Polym. J., 9, 219 (1977).

6) A. Hayashi, K. Kinoshita, M. Kuwano and A. Nose, Polym. J., 10, 485 (1978).

7) A. Hayashi, K. Kinoshita and S. Yasueda, Polym. J., 12, 447 (1980).

8) A. Hayashi, K. Kinoshita, Y. Miyake and C. Cho, Agric. Biol. Chem., 47, 1699 (1983).

9) "Atelocollagen Jyoho," Nos. 1 and 2, Koken, Tokyo, Japan.

10) A. Hayashi, K. Kinoshita and Y. Kotani, Agric. Biol. Chem., 47, 1705 (1983).

11) P. J. Flory, Proc. R. Soc. London, Ser. A, 234, 73 (1956). 